\title{
STABLE DYNAMIC INVERSION OF NONMINIMUM-PHASE SCALAR LINEAR SYSTEMS $^{1}$
}

\author{
Daniele Pallastrelli Aurelio Piazzi \\ Dipartimento di Ingegneria dell'Informazione, University \\ of Parma (Italy), e-mail: dapallas@tin.it, \\ aurelio@ce.unipr.it
}

\begin{abstract}
The paper presents a transfer function approach to the stable dynamic inversion of nonminimum-phase scalar linear systems. The technique is based on the study of the structure of the causal unstable input obtained with the standard inversion technique. It is shown that the unbounded term of this input can be decomposed as the sum of a linear combination of unstable zero modes for which new formulae are provided. Then, a closed-form expression of the bounded noncausal solution of the input-output inversion problem is proposed. An automatic inversion scheme built by exploiting the new inversion formula is also presented. Copyright ${ }^{(0)} 2005$ IFAC
\end{abstract}

Keywords: Feedforward control, inversion, non-minimum phase systems, linear systems, transfer functions.

\section{INTRODUCTION}

In the control theory literature noncausal feedforward based on stable (dynamic) inversion is known to be an effective technique to improve the performances of tracking and regulation (Devasia et al., 1996; Hunt et al., 1996; Piazzi and Visioli, 2001b). The great majority of the techniques on the subject has adopted a purely state-space approach to solve the relevant stable inversion problem for linear and nonlinear systems. An exception to this mainstream course is the work of Ramakrishna et al. (Ramakrishna et al., 2001) which is about the effects of parameter variations on the feedforward input synthesized by stable inversion and uses a transfer function approach in some parts of its formal development. Probably, the main reason of the polarization toward the state-space is the attention to nonlinear problems given by the first researchers of the sub-

\footnotetext{
1 This work was partially supported by MIUR scientific research funds under the framework of the COFIN 2002 projects.
}

ject, notably Devasia, Chen and Paden (Devasia et al., 1996) and Hunt and Meyer (Hunt and Meyer, 1997). However, a purely transfer function approach to the linear problem has its advantages as this article is about to disclose.

The contribution of this article is the solution of the stable inversion problem for nonminimumphase scalar linear systems through explicit formulae. We study the structure of the causal unstable input obtained with the standard inversion technique. It is then shown that the unbounded convolutional integral contained in this input can be decomposed in two noncausal signals: the unbounded and the bounded part. New formulae are given to characterize this unbounded signal as linear combination of unstable zero modes. We therefore solve the stable input-output inversion problem by (mathematically) cancelling the unbounded signal from the standard inversion input. Finally we obtain a closed-form expression of the bounded noncausal input that causes the desired output signal. An automatic inversion system built by exploiting the provided inversion for- 
mula is also presented. Due to lack of space, proofs of the formal statements reported in this article are omitted. They can be found in (Pallastrelli et al., 2004).

Notation. The set of positive reals and the set of complex numbers with positive real part are denoted by $\mathbb{R}_{+}$and $\mathbb{C}_{+}$respectively. We denote by $C^{k}$ the space of the scalar real functions (defined over $\mathbb{R}$ ) that are continuous till the $k$ th time derivative, and by $B C^{k}$ the subset of $C^{k}$ of the functions that are continuous and bounded over $\mathbb{R}$ till the $k$ th time derivative. The $i$ th derivative operator is denoted by $D^{i} . P^{k}$ is the set of $C^{k}$ piecewise continuous functions defined by $P^{k}:=$ $\left\{f: \mathbb{R} \rightarrow \mathbb{R}: \exists\left\{t_{1}, \ldots, t_{n}, \ldots\right\} \subset \mathbb{R} \ni f \in C^{k}(\mathbb{R}-\right.$ $\left.\left\{t_{1}, \ldots, t_{n}, \ldots\right\}\right)$ and $\exists \lim _{t \rightarrow t_{j}^{-}} D^{i} f \exists \lim _{t \rightarrow t_{j}^{+}} D^{i} f$ $j \in \mathbb{N}, i=0, \ldots, k\}$. The Heaviside function is denoted by $\mathbf{1}(t)$.

\section{PRELIMINARIES AND PROBLEM STATEMENT}

Let $\Sigma$ be an $n$ th-order linear, time-invariant, scalar continuous-time system with transfer function

$H(s):=k_{1} \frac{b(s)}{a(s)}=k_{1} \frac{s^{m}+b_{m-1} s^{m-1}+\cdots+b_{1} s+b_{0}}{s^{n}+a_{n-1} s^{n-1}+\cdots+a_{1} s+a_{0}}$.

$H(s)$ is stable, nonminimum phase, and does not have $j$-axis zeroes. In addition, $H(s)$ has no polezero cancellations: $a(s)$ and $b(s)$ are coprime. The input and output of $\Sigma$ are $u \in \mathbb{R}$ and $y \in$ $\mathbb{R}$, respectively. The relative order (or relative degree) of $\Sigma$ is $\rho:=n-m$. Finally, the set of all cause/effect pairs associated with $\Sigma$ is denoted by

$$
\begin{aligned}
\mathcal{B}:=\{ & (u(\cdot), y(\cdot)) \in P^{m} \times P^{n}: \\
& (u(\cdot), y(\cdot)) \text { is a weak solution of } \\
& D^{n} y+a_{n-1} D^{n-1} y+\cdots+a_{0} y= \\
& \left.k_{1}\left(D^{m} u+b_{m-1} D^{m-1} u+\cdots+b_{0} u\right)\right\} .
\end{aligned}
$$

$\mathcal{B}$ is the behaviour set of $\Sigma$ with an input/output partition. The concept of "weak solution" has been rigorously exposed in the book of Polderman and Willems devoted to the behavioral approach to system theory (Polderman and Willems, 1998). Roughly speaking, $(u(\cdot), y(\cdot))$ is a weak solution when there exists a sequence $\left\{u_{i}(\cdot), y_{i}(\cdot)\right\} \in C^{m} \times$ $C^{n}$ satisfying the differential equation of $\Sigma$ and converging to it for $i \rightarrow+\infty$. The following result is useful to introduce our problem:

Proposition 1. (Polderman and Willems, 1998) Consider any pair $(u(\cdot), y(\cdot)) \in \mathcal{B}$. Then given $l$, a nonnegative integer, $u(\cdot) \in C^{l}$ if and only if $y(\cdot) \in C^{\rho+l}$.
Now suppose that we are given an output function $y_{d}: \mathbb{R} \rightarrow \mathbb{R}$ for which we conveniently assume $y_{d}(t)=0$ for $t<0$ and we are interested in finding a control input $u_{d}: \mathbb{R} \rightarrow \mathbb{R}$ which yields the desired output $y_{d}(\cdot)$. Considering the nonminimum-phase property of $\Sigma$, the critical issue is to find a bounded (possibly noncausal) $u_{d}(\cdot)$. Then the Stable Dynamic Input-Output Inversion (SDIOI) Problem can be introduced as it follows:

SDIOI Problem: Let be given any $y_{d}(\cdot) \in B C^{\rho}$ with $y_{d}(t)=0$ for $t<0$. Then find $u_{d}(t) \in B C^{0}$ such that $\left(u_{d}(\cdot), y_{d}(\cdot)\right) \in \mathcal{B}$.

It will turn out (cf. Section 4) that to each bounded output $y_{d}(\cdot)$ there corresponds exactly one bounded input $u_{d}(\cdot)$.

\section{ON THE STRUCTURE OF THE STANDARD INVERSION SOLUTION}

Denote with $Y_{d}(s):=\mathcal{L}\left[y_{d}(t)\right]$ the Laplace transform of $y_{d}(t)$ and, performing the inverse Laplace transformation, compute

$$
u_{u}(t):=\mathcal{L}^{-1}\left[H^{-1}(s) Y_{d}(s)\right] .
$$

Function $u_{u}(\cdot)$ is the result of the standard inputoutput inversion for which $u_{u}(t)=0$ for $t<0$ and evidently $\left(u_{u}(\cdot), y_{d}(\cdot)\right) \in \mathcal{B}$. If $y_{d}(\cdot) \in B C^{\rho}$ then $u_{u}(\cdot) \in C^{0}$ but, due to the unstable zero dynamics, $u_{u}(\cdot) \notin B C^{0}$, i.e. $u_{u}(\cdot)$ is a continuous but unbounded signal, hence it can not be used as a feedforward input for control purposes.

By polynomial division, the inverse of the transfer function (1) can be expressed as

$$
\begin{aligned}
H^{-1}(s) & =\frac{1}{k_{1}} \frac{a(s)}{b(s)} \\
& =\xi_{\rho} s^{\rho}+\xi_{\rho-1} s^{\rho-1}+\cdots+\xi_{0}+H_{0}(s),
\end{aligned}
$$

where $H_{0}(s)$ is a strictly proper rational function representing the zero dynamics of $\Sigma$. By using the fraction expansion, $H_{0}(s)$ can be decomposed into stable and unstable parts according to

$H_{0}(s)=\frac{c(s)}{b(s)}=H_{0}^{-}(s)+H_{0}^{+}(s)=\frac{d(s)}{b^{-}(s)}+\frac{e(s)}{b^{+}(s)}$,

where $b^{-}(s)$ and $b^{+}(s)$ are the monic polynomials containing the roots of $b(s)$ with negative and positive real part, respectively. Moreover, defining $m^{-}:=\operatorname{deg}\left\{b^{-}(s)\right\}, m^{+}:=\operatorname{deg}\left\{b^{+}(s)\right\}$ results in: $\operatorname{deg}\{d(s)\} \leq m^{-}-1$ and $\operatorname{deg}\{e(s)\} \leq m^{+}-1$. The modes associated to $b^{-}(s)$ and $b^{+}(s)$ be denoted by $m_{i}^{-}(t), i=1, \ldots, m^{-}$, and by $m_{i}^{+}(t), i=$ $1, \ldots, m^{+}$respectively. Note that if $z$ is a real zero of $b^{-}(s)$ or $b^{+}(s)$ with multiplicity $h$, then the associated modes are defined by:

$$
\left\{e^{z t}, t e^{z t}, \ldots, t^{h-1} e^{z t}\right\},
$$

whereas if $\sigma \pm j \omega$ are complex zeroes of $b^{-}(s)$ or $b^{+}(s)$ with multiplicity $h$, then the associated modes are 


$$
\begin{aligned}
& \left\{e^{\sigma t} \sin \omega t, e^{\sigma t} \cos \omega t, \ldots\right. \\
& \left.\quad \ldots, t^{h-1} e^{\sigma t} \sin \omega t, t^{h-1} e^{\sigma t} \cos \omega t\right\}
\end{aligned}
$$

Expression (2), (3), and (4) permit writing:

$$
\begin{aligned}
u_{u}(t)= & \mathcal{L}^{-1}\left[\left(\xi_{\rho} s^{\rho}+\xi_{\rho-1} s^{\rho-1}+\cdots+\xi_{0}\right) Y_{d}(s)\right. \\
& \left.+H_{0}^{-}(s) Y_{d}(s)+H_{0}^{+}(s) Y_{d}(s)\right]
\end{aligned}
$$

The three addends contained in the previous expression will be studied in detail below.

The first term is

$$
\begin{aligned}
& \mathcal{L}^{-1}\left[\left(\xi_{\rho} s^{\rho}+\xi_{\rho-1} s^{\rho-1}+\cdots+\xi_{0}\right) Y_{d}(s)\right] \\
& =\xi_{\rho} D^{\rho} y_{d}(t)+\xi_{\rho-1} D^{\rho-1} y_{d}(t)+\cdots+\xi_{0} y_{d}(t)
\end{aligned}
$$

which is continuous and bounded over $\mathbb{R}$ provided that $y_{d}(\cdot) \in B C^{\rho}$.

By defining $\eta^{-}(t)$ the noncausal inverse Laplace transformation of $H_{0}^{-}(s)$ such that $\eta^{-}(t) \mathbf{1}(t)=$ $\mathcal{L}^{-1}\left[H_{0}^{-}(s)\right]$, the second addend of expression $(5)$ can be given by this convolutional integral:

$$
\mathcal{L}^{-1}\left[H_{0}^{-}(s) Y_{d}(s)\right]=\int_{0}^{t} \eta^{-}(t-\tau) y_{d}(\tau) \mathrm{d} \tau .
$$

Integral (6) is the response of a BIBO system (the zero stable dynamics) to the bounded signal $y_{d}(\cdot)$. Hence, it is bounded too, and continuous over $\mathbb{R}$.

By defining $\eta^{+}(t)$ the noncausal inverse Laplace transformation of $H_{0}^{+}(s)$ such that $\eta^{+}(t) \mathbf{1}(t)=$ $\mathcal{L}^{-1}\left[H_{0}^{+}(s)\right]$, the third addend being considered is

$$
\mathcal{L}^{-1}\left[H_{0}^{+}(s) Y_{d}(s)\right]=\int_{0}^{t} \eta^{+}(t-\tau) y_{d}(\tau) \mathrm{d} \tau .
$$

which is, in general, unbounded over $[0,+\infty)$ due to instability of $H_{0}^{+}(s)$, the unstable zero dynamics of $\Sigma$.

Summing the three addends, we obtain the closedform expression of the unstable inverse $u_{u}(t)$ : $(t \in \mathbb{R})$

$$
\begin{gathered}
u_{u}(t)=\xi_{\rho} D^{\rho} y_{d}(t)+\cdots+\xi_{1} D y_{d}(t)+\xi_{0} y_{d}(t) \\
+\int_{0}^{t} \eta^{-}(t-\tau) y_{d}(\tau) \mathrm{d} \tau+\int_{0}^{t} \eta^{+}(t-\tau) y_{d}(\tau) \mathrm{d} \tau
\end{gathered}
$$

In full generality, consider that the unstable zeros of $\Sigma$ are given by $z_{i} \in \mathbb{R}_{+}, i=1, \ldots, l$ with associated multiplicity $m_{i}$ and by $\sigma_{j} \pm \omega_{j} \in \mathbb{C}_{+}$, $j=1, \ldots, h$ with associated multiplicity $m_{j}$. Then, by applying the partial fraction expansion on $H_{0}^{+}(s)$ we obtain:

$$
\begin{aligned}
\eta^{+}(t)= & \sum_{i=1}^{l} \sum_{j=0}^{m_{i}-1} k_{i, j} t^{j} e^{z_{i} t} \\
& +\sum_{i=1}^{h} \sum_{j=0}^{r_{i}-1} t^{j} e^{\sigma_{i} t}\left(p_{i, j} \cos \omega_{i} t+q_{i, j} \sin \omega_{i} t\right)
\end{aligned}
$$

where $k_{i, j}, p_{i, j}, q_{i, j} \in \mathbb{R}$ are appropriate coefficients. The following two propositions are useful in the development.

Proposition 2. Let $h: \mathbb{R} \rightarrow \mathbb{R}$ be defined by

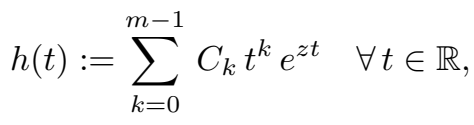

where $m \in \mathbb{N}, z \in \mathbb{R}_{+}$, and $C_{k} \in \mathbb{R}$. Let $y(\cdot) \in B C^{0}$ with $y(t)=0$ for $t<0$. Then $\forall t \in \mathbb{R}$ the convolution between $y(t)$ and $h(t) \mathbf{1}(t)$ can be written as

$$
\begin{aligned}
& y(t) * h(t) \mathbf{1}(t)= \\
& \sum_{k=0}^{m-1} \gamma_{k} t^{k} e^{z t}-\int_{t}^{+\infty} h(t-\tau) y(\tau) \mathrm{d} \tau,
\end{aligned}
$$

where:

$$
\gamma_{k}:=\sum_{i=k}^{m-1} C_{i}\left(\begin{array}{l}
i \\
k
\end{array}\right) \mathcal{L}\left[(-t)^{i-k} y(t)\right]_{s=z} .
$$

Proposition 3. Let $h: \mathbb{R} \rightarrow \mathbb{R}$ be defined by

$$
h(t):=\sum_{k=0}^{m-1} t^{k} e^{\sigma t}\left(p_{k} \cos \omega t+q_{k} \sin \omega t\right) \quad \forall t \in \mathbb{R},
$$

where $m \in \mathbb{N}, \sigma, \omega \in \mathbb{R}_{+}$, and $p_{k}, q_{k} \in \mathbb{R}$. Let $y(\cdot) \in B C^{0}$ with $y(t)=0$ for $t<0$. Then $\forall t \in \mathbb{R}$ the convolution between $y(t)$ and $h(t) \mathbf{1}(t)$ can be written as

$$
\begin{aligned}
y(t) * h(t) \mathbf{1}(t)= & \sum_{k=0}^{m-1} t^{k} e^{\sigma t}\left(\alpha_{k} \cos \omega t+\beta_{k} \sin \omega t\right) \\
& -\int_{t}^{+\infty} h(t-\tau) y(\tau) \mathrm{d} \tau,
\end{aligned}
$$

where:

$\alpha_{k}:=\sum_{i=k}^{m-1}\left(\begin{array}{l}i \\ k\end{array}\right) \mathcal{L}\left[(-t)^{i-k}\left(p_{i} \cos \omega t-q_{i} \sin \omega t\right) y(t)\right]_{s=\sigma}$

$\beta_{k}:=\sum_{i=k}^{m-1}\left(\begin{array}{l}i \\ k\end{array}\right) \mathcal{L}\left[(-t)^{i-k}\left(p_{i} \sin \omega t+q_{i} \cos \omega t\right) y(t)\right]_{s=\sigma}$.

Using the above propositions and the explicit expression of $\eta^{+}(t)$ given by (9) we obtain ( $\forall t \in$ $\mathbb{R})$

$$
\begin{aligned}
& \int_{0}^{t} \eta^{+}(t-\tau) y_{d}(\tau) \mathrm{d} \tau= \\
& f(t)+g(t)-\int_{t}^{+\infty} \eta^{+}(t-\tau) y_{d}(\tau) \mathrm{d} \tau
\end{aligned}
$$

where $f$ and $g$ are defined by:

$$
f(t):=\sum_{i=1}^{l} \sum_{k=0}^{m_{i}-1} \gamma_{i, k} t^{k} e^{z_{i} t}
$$




$$
\begin{aligned}
& \gamma_{i, k}:=\sum_{j=k}^{m_{i}-1} k_{i, j}\left(\begin{array}{l}
j \\
k
\end{array}\right) \mathcal{L}\left[(-t)^{j-k} y_{d}(t)\right]_{s=z_{i}} \\
& g(t):=\sum_{i=1}^{h} \sum_{k=0}^{r_{i}-1} t^{k} e^{\sigma_{i} t}\left(\alpha_{i, k} \cos \omega_{i} t+\beta_{i, k} \sin \omega_{i} t\right) \\
& \alpha_{i, k}:=\sum_{j=k}^{r_{i}-1}\left(\begin{array}{l}
j \\
k
\end{array}\right) \\
& \mathcal{L}\left[y_{d}(t)(-t)^{j-k}\left(p_{i, j} \cos \omega_{i} t-q_{i, j} \sin \omega_{i} t\right)\right]_{s=\sigma_{i}} \\
& \beta_{i, k}:=\sum_{j=k}^{r_{i}-1}\left(\begin{array}{l}
j \\
k
\end{array}\right) \\
& \cdot \mathcal{L}\left[y_{d}(t)(-t)^{j-k}\left(p_{i, j} \sin \omega_{i} t+q_{i, j} \cos \omega_{i} t\right)\right]_{s=\sigma_{i}}
\end{aligned}
$$

This result can be summarized by $(t \in \mathbb{R})$ :

$$
\begin{aligned}
\int_{0}^{t} \eta^{+}(t-\tau) y_{d}(\tau) \mathrm{d} \tau= & \sum_{i=1}^{m^{+}} d_{i} m_{i}^{+}(t) \\
& -\int_{t}^{+\infty} \eta^{+}(t-\tau) y_{d}(\tau) \mathrm{d} \tau,
\end{aligned}
$$

where $d_{i}$ are appropriate coefficients, calculated by means of the above definitions.

Consider now the right side of identity (23). Being $m_{i}^{+}(t), i=1, \ldots, m^{+}$unstable modes, it results that the first terms are not bounded whereas the integral $\int_{t}^{+\infty} \eta^{+}(t-\tau) y_{d}(\tau) \mathrm{d} \tau$ is bounded as stated by the following proposition.

Proposition 4. If $y_{d}(t)$ is bounded over $\mathbb{R}$, then the integral

$$
\int_{t}^{+\infty} \eta^{+}(t-\tau) y_{d}(\tau) \mathrm{d} \tau
$$

is bounded over $\mathbb{R}$ as well.

Using expression (23), the unbounded input $u_{u}(t)$ (8) can be then rewritten as $(t \in \mathbb{R})$ :

$$
\begin{aligned}
u_{u}(t) & =\xi_{\rho} D^{\rho} y_{d}(t)+\cdots+\xi_{1} D y_{d}(t)+\xi_{0} y_{d}(t) \\
& -\int_{t}^{+\infty} \eta^{+}(t-\tau) y_{d}(\tau) \mathrm{d} \tau+\sum_{i=1}^{m^{+}} d_{i} m_{i}^{+}(t) \\
& +\int_{0}^{t} \eta^{-}(t-\tau) y_{d}(\tau) \mathrm{d} \tau
\end{aligned}
$$

\section{SOLUTION OF THE STABLE DYNAMIC INVERSION PROBLEM}

The following lemma points out that any linear combinations of stable and unstable modes in-

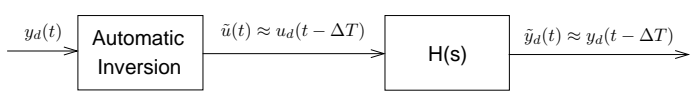

Fig. 1. Automatic inversion schema.

jected on the input of $\Sigma$ results into a null output response.

\section{Lemma 5.}

$\left(\sum_{i=1}^{m^{-}} \mu_{i} m_{i}^{-}(\cdot)+\sum_{j=1}^{m^{+}} \nu_{j} m_{j}^{+}(\cdot), 0\right) \in \mathcal{B} \forall \mu_{i}, \nu_{j} \in \mathbb{R}$

Then, taking into account the structure of the unbounded input (25) we can straightforwardly solve the posed inversion problem.

Theorem 6. (Solution of the SDIOI Problem) Let be given any $y_{d}(\cdot) \in B C^{\rho}$ with $y_{d}(t)=0$ for $t<0$. For all $t \in \mathbb{R}$ define

$$
\begin{aligned}
& u_{d}(t):=\xi_{\rho} D^{\rho} y_{d}(t)+\cdots+\xi_{0} y_{d}(t) \\
& +\int_{0}^{t} \eta^{-}(t-\tau) y_{d}(\tau) \mathrm{d} \tau-\int_{t}^{+\infty} \eta^{+}(t-\tau) y_{d}(\tau) \mathrm{d} \tau
\end{aligned}
$$

then $u_{d}(\cdot)$ is the sole function in $B C^{0}$ satisfying $\left(u_{d}(\cdot), y_{d}(\cdot)\right) \in \mathcal{B}$.

By comparing the solution (26) with expression (23) for the negative times we infer that $u_{d}(t)=$ $-\sum_{i=1}^{m^{+}} d_{i} m_{i}^{+}(t)$ for $t<0$. This makes evident that the solution to the SDIOI problem is a noncausal signal.

Remark 1. Note that by defining:

$$
\eta(t):=\mathbf{1}(t) \eta^{-}(t)-\mathbf{1}(-t) \eta^{+}(t),
$$

the solution (26) can be rewritten:

$$
\begin{aligned}
u_{d}(t) & =\xi_{\rho} D^{\rho} y_{d}(t)+\cdots+\xi_{1} D y_{d}(t)+\xi_{0} y_{d}(t) \\
& +\int_{-\infty}^{+\infty} \eta(t-\tau) y_{d}(\tau) \mathrm{d} \tau .
\end{aligned}
$$

The integral appearing in (28) is the compact closed-form expression of the noncausal bounded output of the zero dynamics driven by the signal $y_{d}(t)$.

\section{AUTOMATIC INVERSION METHOD}

In this section an automatic method to obtain the signal $u_{d}(\cdot)$ through the elaboration of $y_{d}(\cdot)$ applied with a preaction time $\Delta T$ is presented. The method is based on the approximation of the integral $\int_{t}^{+\infty} \eta^{+}(t-\tau) y_{d}(\tau) \mathrm{d} \tau$ that appears in the (26).

In general, the Cavalieri-Simpson can be used to approximate the value of a defined integral as follows. If $\tau_{i}:=i \frac{\Delta T}{n}$ and $n$ is large enough: 


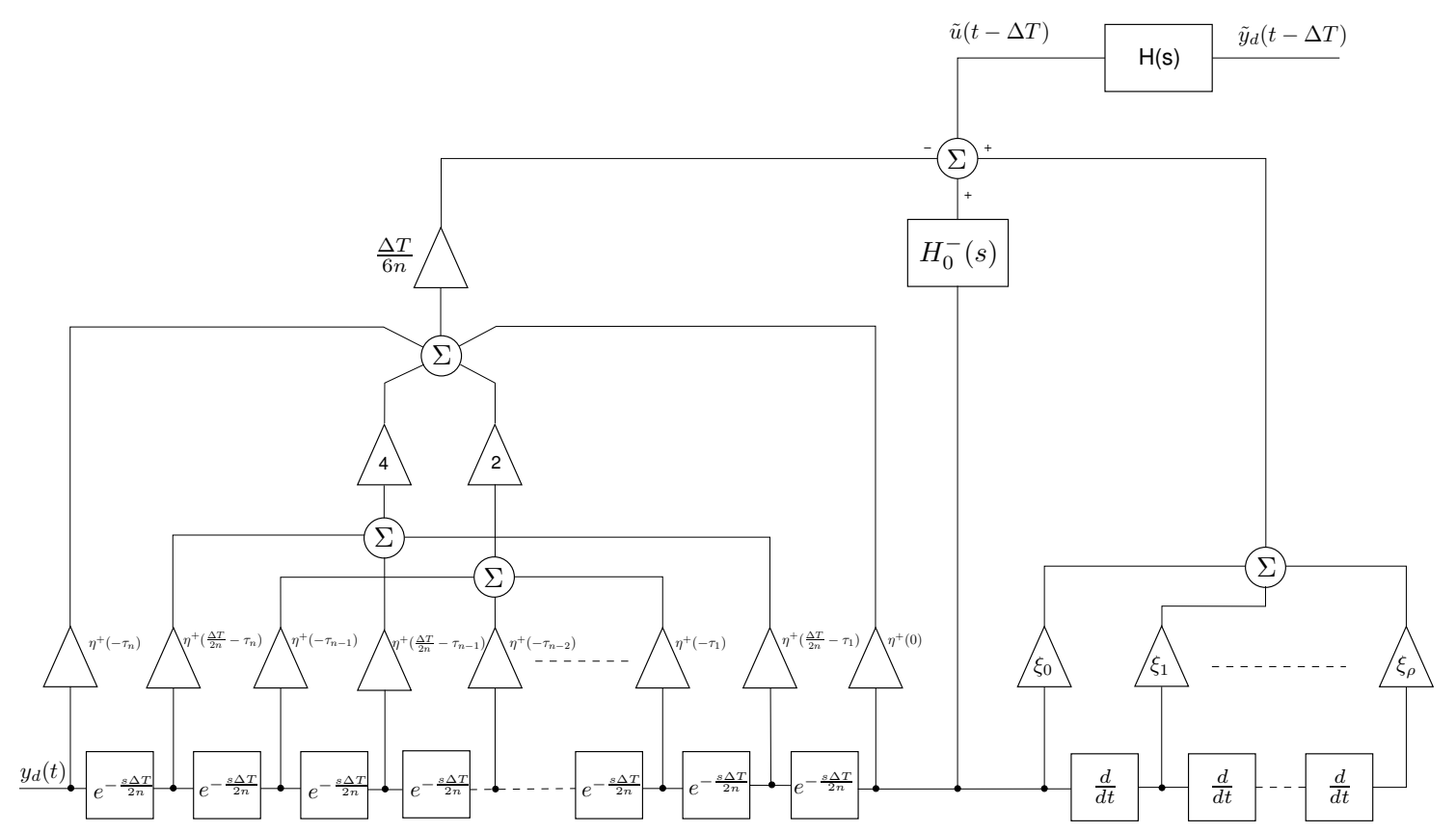

Fig. 2. Automatic inversion generator.

$$
\begin{array}{rl}
\int_{t}^{t+\Delta T} & f(x) \mathrm{d} x \approx \frac{\Delta T}{6 n}\left\{f(t)+f\left(t+\tau_{n}\right)\right. \\
+ & \left.+2 \sum_{i=1}^{n-1} f\left(t+\tau_{i}\right)+4 \sum_{i=1}^{n} f\left(t-\frac{\Delta T}{2 n}+\tau_{i}\right)\right\} .
\end{array}
$$

Under appropriate hypothesis, the method can be applied to the (26), obtaining:

$$
\begin{aligned}
& \int_{t}^{+\infty} \eta^{+}(t-\tau) y_{d}(\tau) \mathrm{d} \tau \approx \int_{t}^{t+\Delta T} \eta^{+}(t-\tau) y_{d}(\tau) \mathrm{d} \tau \\
& \approx \frac{\Delta T}{6 n}\left\{\eta^{+}(0) y_{d}(t)+\eta^{+}\left(-\tau_{n}\right) y_{d}\left(t+\tau_{n}\right)\right. \\
& \quad+2 \sum_{i=1}^{n-1} \eta^{+}\left(-\tau_{i}\right) y_{d}\left(t+\tau_{i}\right) \\
& \left.\quad+4 \sum_{i=1}^{n} \eta^{+}\left(\frac{\Delta T}{2 n}-\tau_{i}\right) y_{d}\left(t-\frac{\Delta T}{2 n}+\tau_{i}\right)\right\}
\end{aligned}
$$

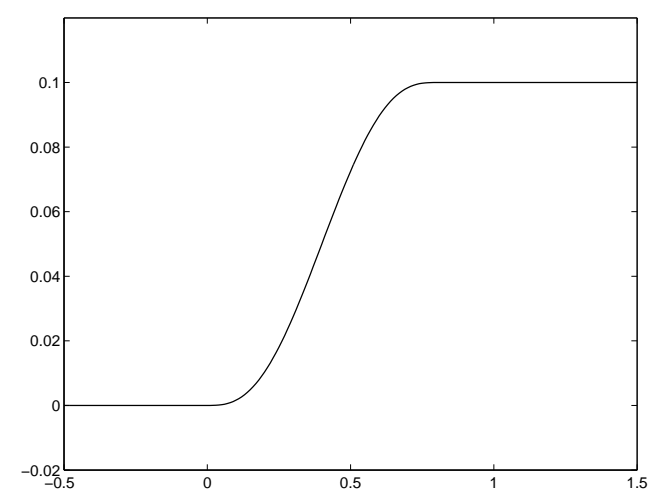

Fig. 3. Desired output: transition polynomial.

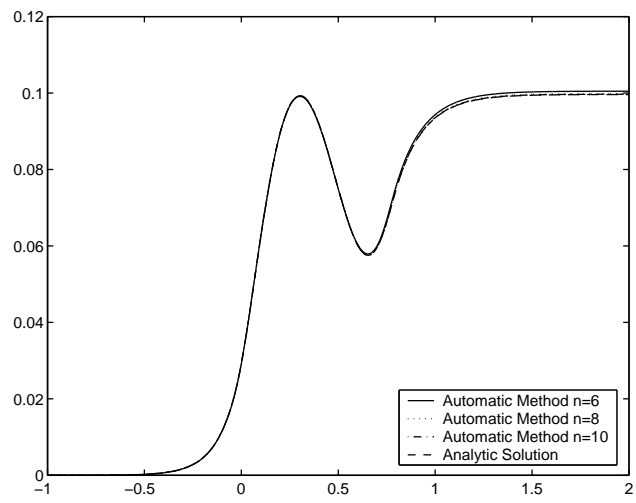

Fig. 4. Input obtained through inversion.

block "Automatic Inversion" of Fig. 1. Indeed the signal $\tilde{u}(t)$ can be automatically generated through the block diagram shown in Fig. 2 employing $2 n$ delay blocks of $\Delta T /(2 n)$ seconds.

\section{EXAMPLE}

As illustrative example, the open-loop end-point control of a flexible link is considered (Piazzi and

Once $H(s)$ (namely the system plant $\Sigma$ ) is fixed, the expression (29) can be used to implement the 


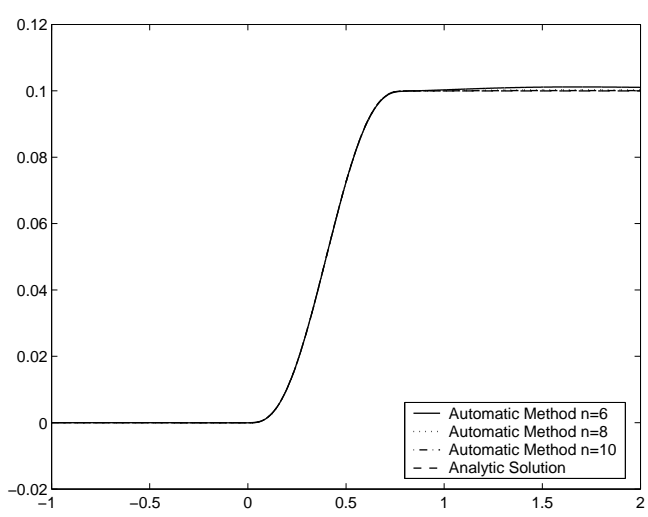

Fig. 5. Output of the system.

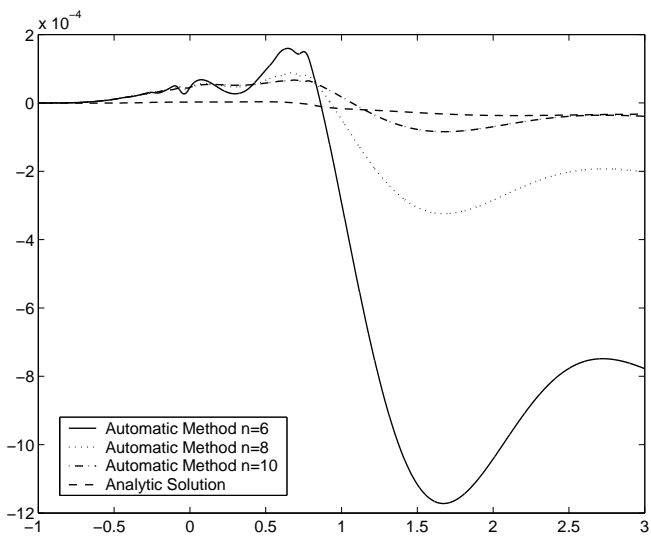

Fig. 6. Error of the inversion.

Visioli, 2001a). The transfer function between the load and the hub angular position is:

$$
H(s):=-0.16 \frac{(s-9.31)(s+6.93)}{(s+1.16)^{2}+2.99^{2}}
$$

and it is nonminimum-phase. The problem is on finding the bounded input signal to apply to the hub to obtain the transition of the load between the initial position $0 \mathrm{rad}$ and the final position 0.1 $\mathrm{rad}$. This is done in 0.8 seconds using the following transition polynomial

$$
y_{d}(t):=0.1\left(6 \frac{t^{5}}{0.8^{5}}-15 \frac{t^{4}}{0.8^{4}}+10 \frac{t^{3}}{0.8^{3}}\right)
$$

valid in the interval $[0,0.8] ; y_{d}(t)=0$ for $t<0$ and $y_{d}(t)=0.1$ for $t>0.8$. Note that $y_{d}(\cdot) \in B C^{2}$ implying the continuity of velocities and accelerations. This desired output function is plotted in Fig. 3. Below, both the analytic solution and the automatic method are applied to this example.

\subsection{Analytic Method}

Using the provided solution (26) along with the closed-form expression (31) of the desired output $y_{d}(t)$ results:

$$
u_{d}(t)= \begin{cases}0.0291 e^{9.31 t} & t<0 \\ 0.204 e^{-6.93 t}-0.0000838 e^{9.31 t} & \\ -0.175+1.687 t-3.63 t^{2} & t \in[0,0.8] \\ +3.05 t^{3}-1.93 t^{4}+1.82 t^{5} & \\ 0.100-6.302 e^{-6.93 t} & t>0.8\end{cases}
$$

The dashed line in Fig. 4 displays the input $u_{d}(t)$ whereas the dashed line in Fig. 5 plots the output signal obtained by simulating the transfer function (30) with the preaction time $\Delta T=1 \mathrm{sec}$.

\subsection{Automatic Inversion}

Three approximation cases have been simulated: $n=6,8,10$ with preaction time $\Delta T=1 \mathrm{sec}$. The figures 4,5 and 6 displays respectively the input, output and error of the automatic inversion, compared with the analytic solution. The comparison between the analytic and automatic methods shows that even with $n=8$ (i.e. with 16 delay blocks) the input and output obtained during the two simulations are practically indistinguishable.

\section{CONCLUSION}

In this paper an explicit formula for the stable inversion of nonminimum-phase scalar linear systems has been presented. Based on this formula, an automatic inversion scheme is proposed to generate an approximation of the bounded noncausal input for a given preaction time.

\section{REFERENCES}

Devasia, S., D. Chen and B. Paden (1996). Nonlinear inversion-based output tracking. IEEE Transactions on Automatic Control AC-41(7), 930-942.

Hunt, L.R. and G. Meyer (1997). Stable inversion for nonlinear systems. Automatica 33(8), 1549-1554.

Hunt, L.R., G. Meyer and R. Su (1996). Noncausal inverses for linear systems. IEEE Transaction on Automatic Control AC-41, 608-611.

Pallastrelli, D., and A. Piazzi (2004). Stable inputoutput inversion of nonminimum-phase scalar linear systems. Technical Report TSC-02/04. University of Parma.

Piazzi, A. and A. Visioli (2001a). End-point control of flexible link via optimal dynamic inversion. In: Proceedings of the 2001 IEEE/ASME International Conference on Advanced Intelligent Mechatronics. Como, Italy. pp. 936-941.

Piazzi, A. and A. Visioli (2001b). Optimal inversion-based control for the set-point regulation of nonminimum-phase uncertain scalar systems. IEEE Transactions on Automatic Control 46(10), 1654-1659.

Polderman, J.W. and J.C. Willems (1998). Introduction to mathematical system theory. Springer. New York, NY.

Ramakrishna, V., L. R. Hunt and G. Meyer (2001). Parameter variations, relative degree, and stable inversion. Automatica 37, 871-880. 